\title{
Design and Synthesis of Fungal-Selective Resorcylate Aminopyrazole Hsp90 Inhibitors
}

David S. Huang, Emmanuelle V. LeBlanc, Tanvi Shekhar-Guturja, Nicole Robbins, Damian J. Krysan, Juan Pizarro, Luke Whitesell*, Leah E. Cowen* and Lauren E. Brown*

* To whom correspondence should be addressed.

Emails: L.W.: luke.whitesell@utoronto.ca; L.E.C.: leah.cowen@utoronto.ca; L.E.B.: brownle@bu.edu

Figure S1

\section{Table of Contents}

Figure S2

Supplemental Table 1

Supplemental Table 2

Supplemental Table 3

Supplemental Table 4

Figure S1. Resorcylate aminopyrazoles with greatest fungal-selectivity in lysates display robust binding to relevant fungal Hsp90 nucleotide-binding domain (NBD).

\section{A) C. neoformans NBD}
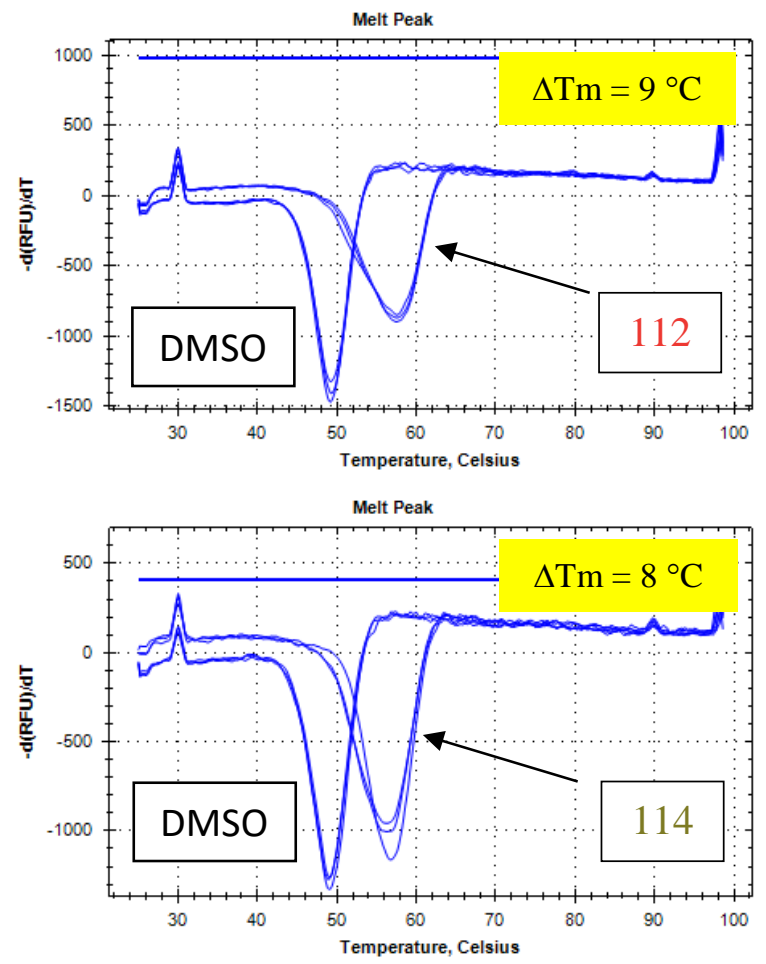

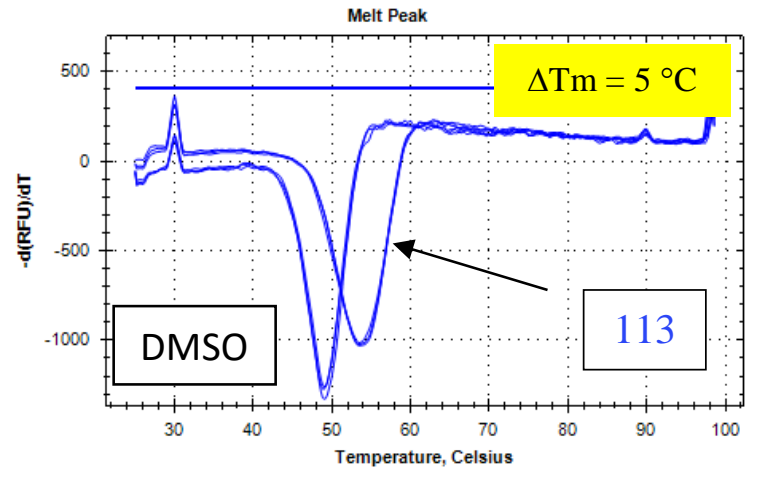

FP Assay

\begin{tabular}{|c|c|}
\hline Compound & Inhibitor Ki (nM) \\
\hline $\mathbf{1 1 2}$ & 3.8 \\
\hline $\mathbf{1 1 3}$ & 19.3 \\
\hline $\mathbf{1 1 4}$ & 5.9 \\
\hline
\end{tabular}



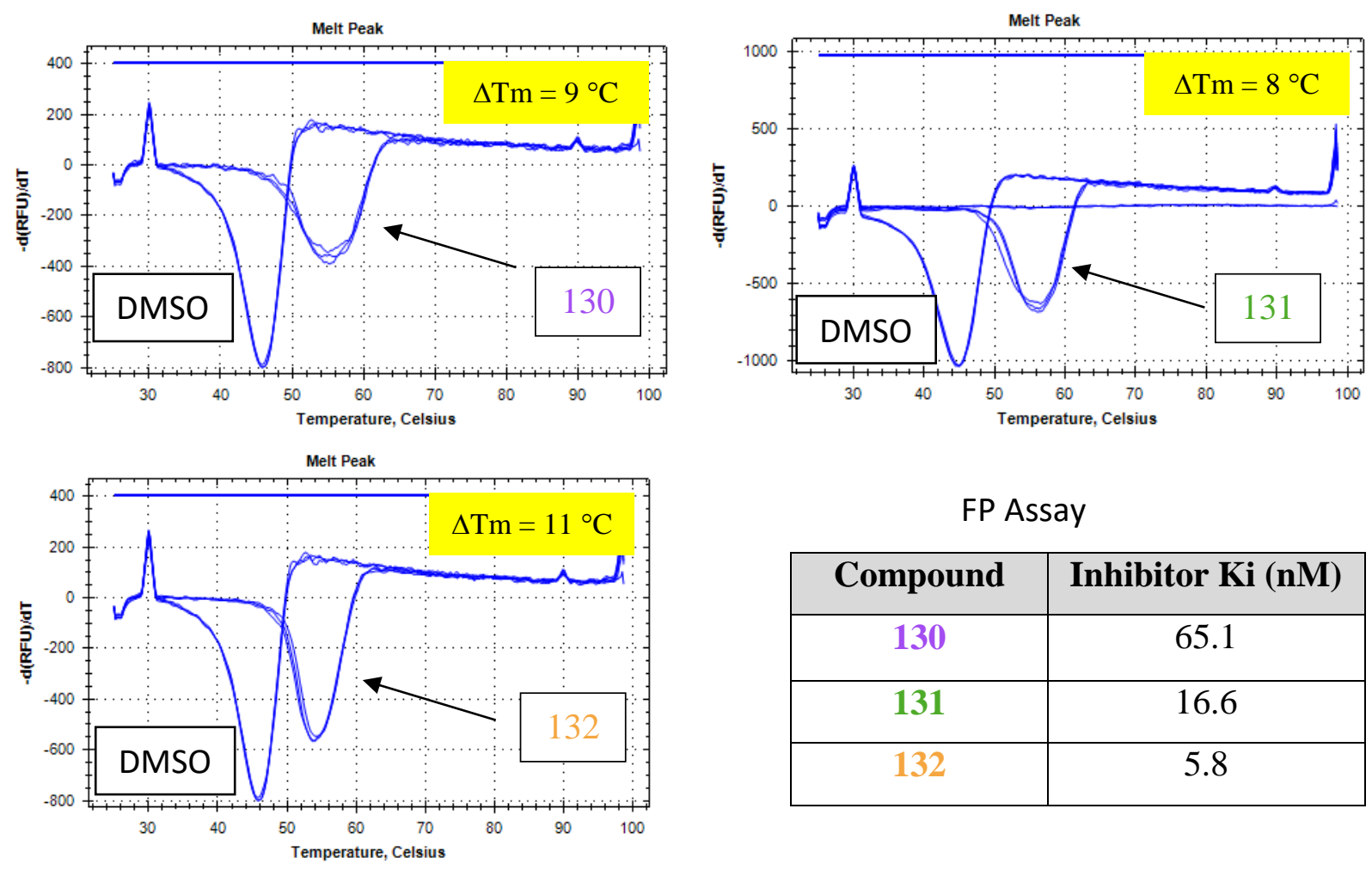

FP Assay

\begin{tabular}{|c|c|}
\hline Compound & Inhibitor Ki (nM) \\
\hline 130 & 65.1 \\
\hline 131 & 16.6 \\
\hline 132 & 5.8 \\
\hline
\end{tabular}

Thermal denaturation curves: (graphs with blue traces). Hsp90 NBDs (A: C. neoformans, B: C. albicans) were incubated with an equimolar concentration of species-selective aminopyrazole or DMSO control, in technical triplicates. The protein was heated from 25 to $98.6^{\circ} \mathrm{C}$ at $0.2^{\circ} \mathrm{C} / 5 \mathrm{~s}$ in the presence of Sypro Orange dye. Traces are plotted as the first derivative of the relative fluorescence signal detected in each well over time. All compounds induce an increase in melting temperature relative to the DMSO control confirming target engagement.

FP: (tables). Binding affinities for each of the three species-selective aminopyrazoles were determined in triplicate wells by equilibrium competition FP assay using C. neoformans and C. albicans Hsp90 NBDs. Ki values were determined as previously described. ${ }^{\mathrm{S} 1-\mathrm{S} 2}$ 
Figure S2. Combination of some non-selective aminopyrazoles with fluconazole generates a fungicidal drug combination against $C$. albicans.

A

\section{Dose-response assays in liquid medium:}

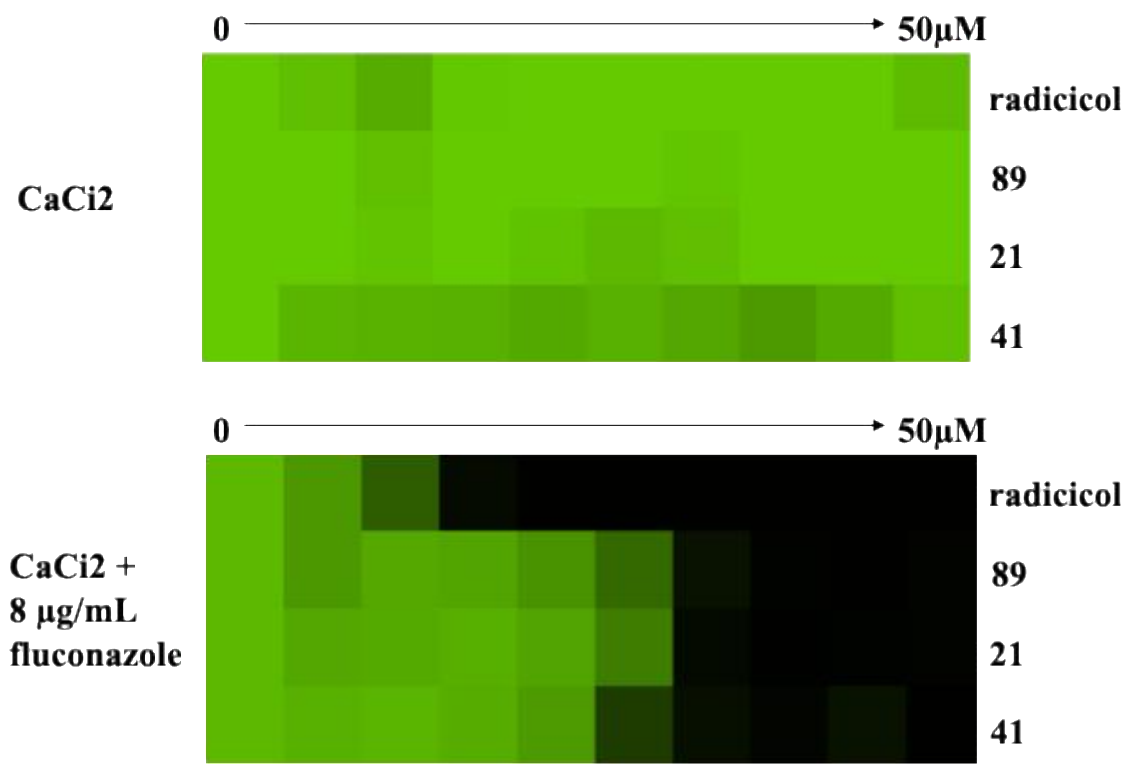

B Survival post exposure (no compound present):
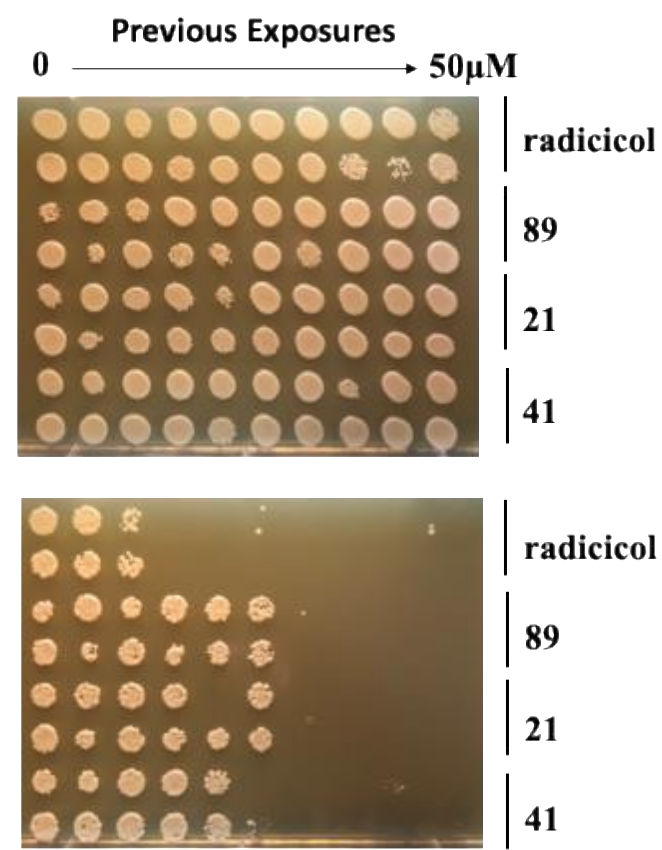

(A) Relative growth inhibition by aminopyrazoles of $C$. albicans clinical isolate CaCi2 in RPMI 1640 medium at $37^{\circ} \mathrm{C}$, with or without a background concentration of $8 \mu \mathrm{g} / \mathrm{mL}$ fluconazole. The effect of 48-hour exposure to inhibitors over a twofold dilution series of concentrations starting at $50 \mu \mathrm{M}$ is displayed in heat-map format. Each colored box represents the mean of technical duplicates. Relative viable cell number was monitored by standard dye reduction assay after 3 -hr incubation with resazurin at $37^{\circ} \mathrm{C}$. (B) Following exposure to compounds, aliquots of the culture in each well were spotted onto compound-free YPD agar and plates incubated at $30{ }^{\circ} \mathrm{C}$ for an additional 24 hours before imaging to assess viability. The entire experiment consisting of growth in liquid culture followed by spotting onto YPD agar was repeated once. 
Supplemental Table 1. FP-based measurement in whole cell lysates of relative Hsp90 binding affinity and fungal selectivity for resorcylate aminopyrazoles.

\begin{tabular}{|c|c|c|c|c|c|c|c|c|}
\hline \multirow[b]{2}{*}{ Compound } & \multicolumn{4}{|c|}{ C. neoformans } & \multicolumn{4}{|c|}{ C. albicans } \\
\hline & Potency $^{a}$ & SEM $^{b}$ & Selectivity ${ }^{c}$ & SEM $^{b}$ & Potency $^{a}$ & SEM $^{b}$ & Selectivity $^{c}$ & SEM \\
\hline 89 & 0.22 & 0.10 & 0.5 & 0.3 & 0.07 & 0.02 & 0.6 & 0.2 \\
\hline 21 & 0.37 & 0.15 & 5.6 & 2.5 & 0.26 & 0.07 & 2.8 & 0.9 \\
\hline 41 & 0.05 & 0.01 & 0.9 & 0.4 & 0.02 & 0.00 & 0.9 & 0.2 \\
\hline 20 & 0.04 & 0.00 & 1.6 & 0.2 & 0.02 & 0.00 & 1 & 0.2 \\
\hline 40 & 0.07 & 0.00 & 1.5 & 0.1 & 0.03 & 0.00 & 1.1 & 0.1 \\
\hline 52 & 0.02 & 0.00 & 1.7 & 0.3 & 0.01 & 0.00 & 1.3 & 0.3 \\
\hline 65 & 0.03 & 0.01 & 2.9 & 1.3 & 0.02 & 0.00 & 1.6 & 0.6 \\
\hline 91 & 0.08 & 0.00 & 16.1 & 3.1 & 0.63 & 0.09 & 0.7 & 0.2 \\
\hline 92 & 0.23 & 0.03 & 11.6 & 3.6 & 1.35 & 0.28 & 0.7 & 0.3 \\
\hline 94 & 0.43 & 0.03 & 26 & 5.6 & 1.61 & 0.36 & 2.5 & 0.9 \\
\hline 98 & 0.20 & 0.03 & 7.7 & 2.7 & 0.37 & 0.08 & 1.5 & 0.6 \\
\hline 106 & 0.06 & 0.00 & 24.2 & 5.1 & 0.64 & 0.04 & 0.8 & 0.2 \\
\hline 108 & 0.07 & 0.01 & 20.6 & 5.1 & 0.40 & 0.00 & 1.2 & 0.2 \\
\hline 111 & 0.15 & 0.01 & 58.7 & 15.4 & 0.98 & 0.17 & 3.1 & 1.1 \\
\hline 112 & 0.33 & 0.04 & 34.4 & 9.2 & 1.95 & 0.12 & 2.1 & 0.4 \\
\hline 113 & 0.98 & 0.14 & 14.3 & 2.1 & 6.17 & 0.42 & 0.8 & 0.1 \\
\hline 114 & 0.26 & 0.03 & 40.7 & 12.2 & 2.19 & 0.10 & 1.7 & 0.4 \\
\hline 115 & 3.49 & 0.49 & 3.6 & 0.7 & 2.13 & 0.08 & 2.1 & 0.2 \\
\hline 116 & 1.40 & 0.18 & 7.9 & 2.2 & 0.77 & 0.05 & 5.2 & 1.1 \\
\hline 117 & 0.42 & 0.06 & 13 & 3.8 & 0.35 & 0.02 & 5.6 & 1.1 \\
\hline 118 & 0.84 & 0.17 & 12.5 & 4.3 & 1.20 & 0.07 & 3.1 & 0.6 \\
\hline 121 & 0.78 & 0.12 & 12.5 & 4.3 & 1.35 & 0.06 & 2.6 & 0.6 \\
\hline 122 & 4.52 & 1.10 & 2.9 & 0.8 & 4.62 & 1.03 & 1 & 0.3 \\
\hline 123 & 0.67 & 0.07 & 16 & 4.5 & 1.08 & 0.21 & 3.6 & 1.3 \\
\hline 130 & 5.76 & 0.47 & 2 & 0.4 & 0.68 & 0.16 & 6.1 & 2.1 \\
\hline 131 & 2.22 & 0.40 & 3.4 & 1.2 & 0.20 & 0.05 & 13.2 & 5.2 \\
\hline 132 & 0.64 & 0.09 & 4.8 & 1.7 & 0.07 & 0.02 & 16.2 & 7 \\
\hline
\end{tabular}

${ }^{\text {a }}$ Mean $\mathrm{EC}_{50}(\mu \mathrm{M})$ : Concentration resulting in 50\% reduction in maximal polarization signal determined in 3 independent equilibrium competition binding experiments each consisting of duplicate determinations b Standard error of the mean

${ }^{\mathbf{c}}$ Mean ratio of $\mathrm{EC}_{50}$ values $(\mu \mathrm{M})$ determined in lysate of human HepG2 cells/indicated fungal species. Results are the mean of 3 independent experiments in each lysate type, each experiment consisting of duplicate determinations 
Supplemental Table 2. FP-based Ki determinations and protein thermal shift measurements for aminopyrazoles with high- and low-potency in whole cell lysates.

\begin{tabular}{|c|c|c|c|c|}
\hline Compound number & $\begin{array}{c}\text { C. neoformans } \\
\text { NBD Ki }(\mathbf{n M})\end{array}$ & $\begin{array}{c}\text { C. neoformans } \\
\text { NBD } \Delta \mathbf{T m}\left({ }^{\circ} \mathbf{C}\right)\end{array}$ & $\begin{array}{c}\text { C. albicans NBD } \\
\mathbf{K i}(\mathbf{n M})\end{array}$ & $\begin{array}{c}\text { C. albicans NBD } \\
\Delta \mathbf{T m}\left({ }^{\circ} \mathbf{C}\right)\end{array}$ \\
\hline $\mathbf{9 1}$ & 1.6 & 11 & 13 & 10 \\
\hline $\mathbf{1 0 0}$ & 2.9 & 11 & 8.5 & 9 \\
\hline $\mathbf{1 0 6}$ & 0.8 & 12 & 9.0 & 11 \\
\hline $\mathbf{1 2 4}$ & 190.4 & 1 & 45.9 & 10 \\
\hline $\mathbf{1 2 7}$ & 311.7 & 0 & 28.9 & 12 \\
\hline
\end{tabular}

Ki: Binding affinities for each of the three species-selective aminopyrazoles were determined in duplicate wells by equilibrium competition FP assay using $C$. neoformans and $C$. albicans Hsp90 NBDs. Ki values were determined as previously described ${ }^{\mathrm{S} 1-\mathrm{S} 2}$.

$\Delta$ Tm: As for Supplemental Figure 1, C. neoformans or C. albicans Hsp90 NBD was combined with equimolar concentrations of the indicated bioactive inhibitors $(10 \mu \mathrm{M})$ or DMSO control, in technical triplicates. The protein was heated from 25 to $98.6^{\circ} \mathrm{C}$ at $0.2{ }^{\circ} \mathrm{C} / 5 \mathrm{~s}$ in the presence of Sypro Orange dye. Increase in melting temperature relative to the DMSO control was reported as $\Delta \mathrm{Tm}$.

Supplemental Table 3. Measurement of binding affinities by SPR

\begin{tabular}{|c|c|c|c|c|c|}
\hline Compound & $\begin{array}{c}\text { Biacore KD } \\
(\mathbf{n M})\end{array}$ & $K_{a}(1 / M \times s)$ & $K_{d}(1 / s)$ & Selectivity & Species \\
\hline 112 & 126 & $1.38 \mathrm{E}+04$ & $2.79 \mathrm{E}-03$ & 1.8 & \multirow{7}{*}{ C. albicans } \\
\hline 113 & 211 & $2.27 \mathrm{E}+04$ & $7.27 \mathrm{E}-03$ & 1.0 & \\
\hline 114 & 86 & $5.62 \mathrm{E}+04$ & $3.99 \mathrm{E}-03$ & 2.9 & \\
\hline 130 & 81 & $1.08 \mathrm{E}+04$ & $2.86 \mathrm{E}-04$ & 13.3 & \\
\hline 131 & 24 & $1.23 \mathrm{E}+04$ & $7.24 \mathrm{E}-04$ & 3.6 & \\
\hline 132 & 9 & $1.56 \mathrm{E}+04$ & $2.31 \mathrm{E}-04$ & 3.4 & \\
\hline Radicicol & 2 & $1.87 \mathrm{E}+05$ & $5.75 \mathrm{E}-04$ & 0.3 & \\
\hline 112 & 74 & $7.02 \mathrm{E}+03$ & $5.16 \mathrm{E}-04$ & 5.0 & \multirow{7}{*}{ C. neoformans } \\
\hline 113 & 67 & $6.21 \mathrm{E}+04$ & $4.18 \mathrm{E}-03$ & 4.7 & \\
\hline 114 & 22 & $6.31 \mathrm{E}+04$ & $1.40 \mathrm{E}-03$ & 9.2 & \\
\hline 130 & 7 & $2.31 \mathrm{E}+04$ & $1.71 \mathrm{E}-04$ & 47.8 & \\
\hline 131 & 380 & $1.15 \mathrm{E}+04$ & $4.38 \mathrm{E}-03$ & 0.6 & \\
\hline 132 & 214 & $9.78 \mathrm{E}+03$ & $2.09 \mathrm{E}-03$ & 0.2 & \\
\hline Radicicol & 2 & $1.03 \mathrm{E}+05$ & $2.32 \mathrm{E}-04$ & 0.4 & \\
\hline & & & $115 \mathrm{E} 02$ & & \multirow{8}{*}{ Human } \\
\hline 112 & 505 & $3.14 \mathrm{E}+03$ & 1.15E-U3 & & \\
\hline$\frac{113}{114}$ & 314 & $1.55 \mathrm{E}+04$ & $4.8 / E-03$ & & \\
\hline $\begin{array}{l}114 \\
130\end{array}$ & 203 & $1.45 \mathrm{E}+\mathrm{U} 4$ & $2.95 \mathrm{E}-03$ & & \\
\hline $\begin{array}{l}130 \\
131\end{array}$ & 354 & Not done & Not done & & \\
\hline$\frac{131}{132}$ & 210 & $5.01 \mathrm{E}+03$ & $1.05 \mathrm{E}-03$ & & \\
\hline 132 & 51 & $7.59 \mathrm{E}+03$ & 3.89E-04 & & \\
\hline Radicicol & 1 & $1.66 \mathrm{E}+05$ & 1.35E-04 & & \\
\hline
\end{tabular}


Supplemental Table 4. Full microsomal stability testing results. Assays performed at Charles River Laboratories (Worcester, MA).

\begin{tabular}{|c|c|c|c|c|c|}
\hline Compound & $\begin{array}{c}\mathbf{T}_{\mathbf{1} / \mathbf{2}} \\
(\mathbf{m i n})\end{array}$ & $\begin{array}{c}\text { CLint } \\
(\mathbf{m L} / \mathbf{m i n} / \mathbf{k g})\end{array}$ & $\begin{array}{c}\text { \% } \\
\text { remaining } \\
\text { at 15 min }\end{array}$ & $\begin{array}{c}\text { \% } \\
\text { remaining } \\
\text { at 60 min }\end{array}$ & $\begin{array}{c}\text { remaining } \\
\text { at 15 min } \\
\text { (NADPH-free) }\end{array}$ \\
\hline $\mathbf{8 3}$ & 3.1 & 1768 & $3.4 \%$ & $1.8 \%$ & $109.1 \%$ \\
\hline $\mathbf{1 0 5}$ & 2.7 & 1989 & $2.3 \%$ & $0 \%$ & $103.6 \%$ \\
\hline $\mathbf{1 1 1}$ & 9.4 & 582 & $20.2 \%$ & $1.8 \%$ & $115.3 \%$ \\
\hline $\mathbf{1 1 2}$ & 3.2 & 1735 & $3.7 \%$ & $0 \%$ & $99.3 \%$ \\
\hline $\mathbf{1 1 3}$ & 3.4 & 1588 & $4.9 \%$ & $0 \%$ & $109.2 \%$ \\
\hline $\mathbf{1 1 4}$ & 17.2 & 318 & $40.1 \%$ & $7.0 \%$ & $108.2 \%$ \\
\hline $\mathbf{1 2 9}$ & 6.4 & 847 & $21.1 \%$ & $0.5 \%$ & $97.9 \%$ \\
\hline $\mathbf{1 3 1}$ & 14.1 & 387 & $35.5 \%$ & $3.9 \%$ & 104.1 \\
\hline $\mathbf{1 3 2}$ & 31.0 & 176 & $63.0 \%$ & $20.6 \%$ & $71.7 \%$ \\
\hline
\end{tabular}

Supplemental Table 5. Aminopyrazoles with bioactivity against $C$. neoformans or $C$. albicans increase thermal stability of the respective recombinant Hsp90 NBD.

\begin{tabular}{|c|c|c|}
\hline Fungal NBD & Compound number & $\Delta \mathbf{T m}\left({ }^{\circ} \mathbf{C}\right)$ \\
\hline \multirow{4}{*}{ C. neoformans } & $\mathbf{2 1}$ & 14 \\
\cline { 2 - 3 } & $\mathbf{2 7}$ & 12 \\
\cline { 2 - 3 } & $\mathbf{2 9}$ & 14 \\
\cline { 2 - 3 } & $\mathbf{4 9}$ & 14 \\
\cline { 2 - 3 } & $\mathbf{8 9}$ & 12 \\
\cline { 2 - 3 } & $\mathbf{1 0 6}$ & 12 \\
\hline \multirow{5}{*}{ C. albicans } & $\mathbf{2 1}$ & 14 \\
\cline { 2 - 3 } & $\mathbf{4 1}$ & 18 \\
\cline { 2 - 3 } & $\mathbf{8 9}$ & 13 \\
\cline { 2 - 3 } & $\mathbf{1 3 0}$ & 9 \\
\hline
\end{tabular}

C. neoformans or $C$. albicans Hsp90 NBD was combined with equimolar concentrations of the indicated bioactive inhibitors $(10 \mu \mathrm{M})$ or DMSO control, in technical triplicates. The protein was heated from 25 to 98.6 ${ }^{\circ} \mathrm{C}$ at $0.2{ }^{\circ} \mathrm{C} / 5 \mathrm{~s}$ in the presence of Sypro Orange dye. All compounds induce an increase in melting temperature relative to the DMSO control, reported as $\Delta \mathrm{Tm}$.

\section{References}

S1. Rossi, A. M.; Taylor, C. W., Analysis of protein-ligand interactions by fluorescence polarization. Nat. Protoc. 2011, 6 (3), 365-87.

S2. Whitesell, L.; Robbins, N.; Huang, D. S.; McLellan, C. A.; Shekhar-Guturja, T.; LeBlanc, E. V.; Nation, C. S.; Hui, R.; Hutchinson, A.; Collins, C.; Chatterjee, S.; Trilles, R.; Xie, J. L.; Krysan, D. J.; Lindquist, S.; Porco, J. A.; Tatu, U.; Brown, L. E.; Pizarro, J.; Cowen, L. E., Structural basis for species-selective targeting of Hsp90 in a pathogenic fungus. Nat. Commun. 2019, 10 (1), 402. 